\section{Des interactions entre bactéries du microbiote pour compenser les effets de la malnutrition chez la drosophile}

Émilie Chabert
École normale supérieure de Lyon, Département de biologie, Master Biosciences, Lyon, France.

emilie.chabert@ens-lyon.fr
> Chez les animaux, la croissance est caractérisée par une prise de poids et une augmentation de la taille de l'individu. Outre les déterminants génétiques, l'apport nutritionnel lors de la phase juvénile est essentiel au bon déroulé de la croissance. Chez l'homme, la malnutrition pendant l'enfance conduit à de très graves séquelles, notamment des retards de croissance et des problèmes cognitifs $[1,2]$. De plus, de récentes études ont démontré que le microbiote intestinal, correspondant à l'ensemble des bactéries colonisant le tractus digestif, jouerait un rôle majeur dans le bon déroulé de la croissance. En effet, l'inoculation du microbiote d'enfants malnutris dans des souris initialement sans microbiote perturbe la croissance et conduit à des anomalies de la structure des os et des fonctions métaboliques de certains organes [3]. Au contraire, l'introduction de certaines souches de Lactobacillus plantarum maintient une croissance normale malgré une alimentation pauvre chez la souris et la drosophile $[4,5]$. Cependant, l'étude du microbiote est rendue difficile par l'abondance des espèces bactériennes dans le tractus intestinal. Ces bactéries forment un écosystème comprenant des réseaux métaboliques complexes. Comprendre les paramètres qui influencent spécifiquement la croissance n'est donc pas tâche aisée.

La drosophile est un organisme modèle particulièrement adapté à l'étude des interactions microbiote/hôte. En effet, elle possède un microbiote simple composé d'une dizaine d'espèces bactériennes seulement. Il est également facile de produire des lignées de drosophiles axéniques, c'est-à-dire sans microbiote, puis de les coloniser avec des souches bactériennes définies.

Pour essayer de mieux comprendre les mécanismes moléculaires des interactions entre le microbiote et l'hôte, Consuegra et al. [6] ont analysé la croissance de la larve de drosophile associée à ses deux bactéries commensales majoritaires: Lactobacillus plantarum et Acetobacter pomorum, dans des conditions de carence nutritive en acides aminés.

Le « dialogue métabolique » entre Lactobacillus plantarum et Acetobacter pomorum favorise l'accroissement de leurs biomasses respectives

Le cycle de vie de la drosophile dure 10 jours. L'embryon se développe en larve et grandit: cette étape correspond à la croissance juvénile. La larve forme ensuite une pupe et se métamorphose en adulte. Une carence nutritive chez une larve conduit à un retard de la métamorphose et à une taille réduite. L'inoculation de $L$. plantarum et $A$. pomorum dans l'intestin d'une larve axénique (sans microbiote) restaure une croissance similaire à celle observée en cas de régime alimentaire normal, ce qui n'est pas le cas lorsque les souches bactériennes sont introduites individuellement. Cette observation démontre la nécessité d'échanges métaboliques entre les deux espèces bactériennes pour avoir un effet bénéfique chez I'hôte. Les auteurs ont donc par la suite caractérisé les interactions entre les deux espèces bactériennes.

Les auteurs ont tout d'abord observé en culture liquide et dans l'intestin de la larve une augmentation de la biomasse des deux populations bactériennes en coculture par rapport aux monocultures. Ces observations suggèrent des échanges métaboliques bénéfiques entre les deux populations. Pour comprendre leurs interactions, les activités métaboliques des deux populations ont été analysées. Précédemment, les auteurs avaient démontré que $A$. pomorum est prototrophe, c'est-à-dire capable de fabriquer toutes les molécules organiques nécessaires à sa survie, alors que L. plantarum a besoin de capter certains acides aminés (arginine, isoleucine, leucine, valine, cystéine) et deux vitamines $B$ (biotine et pantothénate) dans son milieu [7]. Dans cette nouvelle étude, les auteurs sont allés plus loin en montrant que L. plantarum cocultivés avec $A$. pomorum est capable de se multiplier dans un milieu dépourvu des éléments pour lesquels la souche est auxotrophe. Ainsi, A. pomorum favorise la croissance de la population de L. plantarum en lui fournissant acides aminés et vitamines (Figure 1, flèche 1).

Réciproquement, les auteurs ont supposé que l'effet bénéfique de L. plantarum sur la biomasse d'A. pomorum pourrait passer par la production de sous-produits sécrétés par L. plantarum. En accord avec cette hypothèse, l'ajout du surnageant de $L$. plantarum dans une culture de $A$. pomorum augmente la croissance bactérienne. Consuegra et al. ont ensuite démontré que le lactate (ou acide lactique), produit 


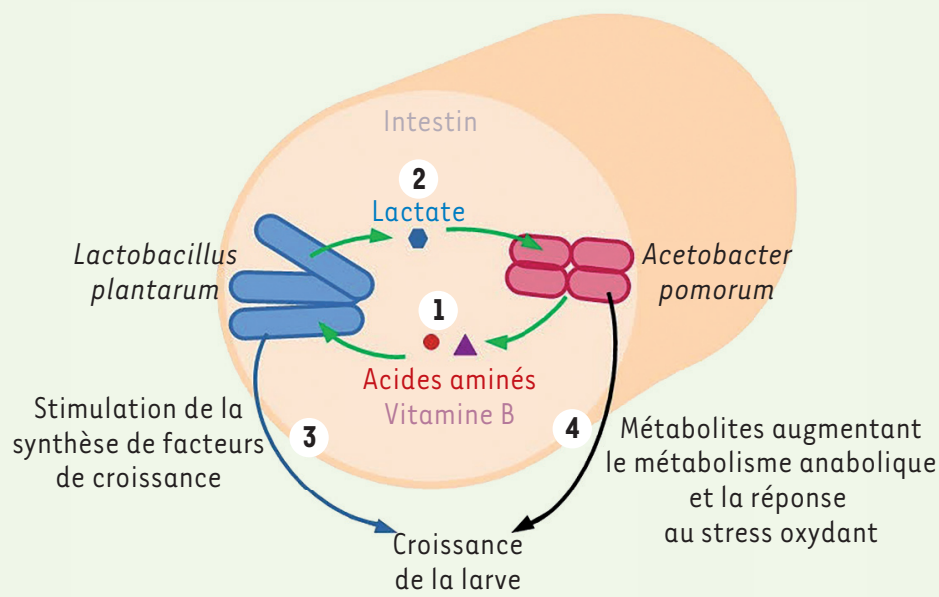

Figure 1. Lactobacillus plantarum et Acetobacter pomorum limitent les effets délétères d'une alimentation pauvre en acides aminés chez la larve de drosophile. L. plantarum et $A$. pomorum interagissent au sein de l'intestin de la larve. 1. L. plantarum importe les acides aminés sécrétés par A. pomorum. 2. A. pomorum utilise le lactate produit par L. plantarum comme source additionnelle de carbone. Les interactions entre les deux bactéries bénéficient à l'hôte. 3. L. plantarum stimule la production de facteurs de croissance chez la larve. 4. A. pomorum fournit des métabolites impliqués dans l'anabolisme et la réponse au stress oxydant de la larve.

majoritaire de la fermentation de L. plantarum, stimulait la croissance d'A. pomorum (Figure 1, flèche 2). En effet, $A$. pomorum importe le lactate extracellulaire et l'utilise comme source de carbone pour faire fonctionner différentes voies de biosynthèse. En particulier, la consommation de lactate par les bactéries $A$. pomorum augmente leur production et leur sécrétion d'acides aminés, dont certains sont nécessaires à la croissance de L. plantarum. Au final, les auteurs ont mis en lumière des interactions aboutissant à un bénéfice réciproque via l'échange de métabolites, conduisant à une augmentation de la biomasse de ces deux populations bactériennes.

\section{Les interactions bénéfiques entre les deux espèces bactériennes modèrent les effets délétères de la malnutrition chez l'hôte} Consuegra et al. ont ensuite cherché à comprendre comment les interactions décrites plus haut pouvaient bénéficier à la larve face aux carences nutritives. Une étude précédente avait démontré que les bactéries du microbiote pouvaient directement servir de source de nutri- ments à la drosophile [8]. Par conséquent, les auteurs ont d'abord proposé que la biomasse bactérienne supplémentaire produite lors de l'interaction entre $L$. plantarum et $A$. pomorum pourrait servir de source de nutriments pour l'hôte et compenser ses carences nutritionnelles. Pour tester cette hypothèse, les auteurs ont inoculé les larves de drosophiles avec des bactéries mortes et ont ensuite soumis ces larves à un stress nutritif. Une légère amélioration de la croissance a été observée par comparaison aux drosophiles sans microbiote. Par conséquent, L. plantarum et $A$. pomorum constituent bien une ressource alimentaire pour l'hôte, mais ces observations indiquent que les bactéries doivent être métaboliquement actives pour exercer un effet complet.

Les auteurs ont ensuite supposé que les acides aminés sécrétés par $A$. pomorum en présence de lactate pourraient être directement absorbés par la larve et constituer une source de nutriments supplémentaire. Les acides aminés produits par $A$. pomorum en absence ou en présence de lactate ont été identifiés et quantifiés par chromatographie en phase liquide à haute performance, et les larves ont été nourries avec ces mélanges d'acides aminés. L'injection dans une drosophile axénique du mélange d'acides aminés sécrétés par $A$. pomorum en présence de lactate n'a pas d'effet positif sur la croissance. Cependant, lorsqu'on fournit à une drosophile inoculée avec L. plantarum le même mélange d'acides aminés, on observe une restauration de la croissance malgré l'alimentation pauvre. Ainsi, les acides aminés produits par $A$. pomorum en présence de lactate ne bénéficient pas directement à l'hôte, mais accentuent l'activité bénéfique de L. plantarum sur l'hôte (Figure 1, flèche 3). Cette activité a été détaillée dans une publication précédente et consiste à augmenter la production de facteurs de croissance appelés DILP (Drosophila insulin-like peptides), des peptides analogues aux IGF (insulin growth factors) des mammifères [5].

Une larve colonisée par $A$. pomorum seule et nourrie avec du lactate a une croissance accélérée par rapport à une larve axénique. Ainsi, A. pomorum a également un effet positif sur l'hôte, dépendant du lactate mais indépendant de L. plantarum. L'hypothèse des auteurs est que cet effet serait dépendant de l'utilisation du lactate par A. pomorum. Pour tester cette hypothèse, les auteurs ont inoculé chez la drosophile des souches mutantes de $A$. pomorum incapables d'utiliser le lactate et ont montré qu'aucun effet bénéfique sur l'hôte n'était observé. Les auteurs ont ensuite déterminé les métabolites spécifiquement produits par $A$. pomorum en présence de lactate, par chromatographie en phase liquide à haute performance et spectrométrie de masse. Au total, 45 métabolites ont été identifiés. Parmi eux, sont présentes la cystéinylglycine et la spermidine, deux métabolites impliqués dans la résistance au stress oxydant chez I'hôte. A. pomorum libère aussi des sousproduits impliqués dans différentes voies anaboliques de l'hôte comme des ribonucléotides ou des cofacteurs d'enzymes [9]. Ces métabolites pourraient constituer un apport nutritionnel supplémentaire pour la larve et l'aider à maintenir une 
croissance normale malgré la malnutrition (Figure 1, flèche 4). D'autres études seront nécessaires pour identifier le rôle précis de ces métabolites sur le métabolisme de l'hôte.

\section{Conclusion}

Le modèle simplifié de microbiote présenté dans cet article met en lumière une coopération trophique étroite entre les bactéries de l'intestin chez la drosophile. Ce dialogue métabolique assure aux deux partenaires une multiplication optimale et, par conséquent, leur offre un avantage pour coloniser efficacement l'intestin de l'hôte. Les auteurs mettent aussi en lumière la façon dont l'hôte profite des sous-produits sécrétés par les bactéries pour combler les potentielles carences de son régime alimentaire et retrouver ainsi une croissance normale. Au vu des effets spectaculaires de l'association de Lactobacillus plantarum et Acetobacter pomorum chez la drosophile, on peut légitimement s'interroger sur leur impact en physio- logie humaine. Les effets bénéfiques observés en cas de malnutrition pourraient être atténués chez l'homme car contrairement aux expériences présentées précédemment, la flore intestinale humaine est constituée de bien plus de deux espèces bactériennes. Des études chez des mammifères comme la souris pourraient apporter des premiers éléments de réponse sur l'utilité potentielle de ces bactéries en tant que probiotiques. $\diamond$

Interaction between intestinal bacteria compensates the deleterious effects of undernutrition in Drosophila

\section{LIENS D'INTÉRÊT}

L'auteure déclare n'avoir aucun lien d'intérêt concernant les données publiées dans cet article.

\section{RÉFÉRENCES}

1. Dewey KG, Begum K. Long-term consequences of stunting in early life. Matern Child Nutr 2011 ; 7 (suppl 3) : 5-18.

2. Goyal MS, Venkatesh S, Milbrandt J, et al. Feeding the brain and nurturing the mind: Linking nutrition and the gut microbiota to brain development. Proc Natl Acad Sci USA 2015 ; 112 : 14105-12.
3. Blanton LV, Charbonneau MR, Salih T, et al. Gut bacteria that prevent growth impairments transmitted by microbiota from malnourished children. Science 2016 ; 351 : aad3311.

4. Schwarzer M, Makki K, Storelli G, et al. Lactobacillus plantarum strain maintains growth of infant mice during chronic undernutrition. Science 2016 ; 351 : 854-7.

5. Storelli G, Defaye A, Erkosar B, et al. Lactobacillus plantarum promotes Drosophila systemic growth by modulating hormonal signals through TOR-dependent nutrient sensing. Cell Metab $2011 ; 14$ : 403-14.

6. Consuegra J, Grenier T, Akherraz H, et al. Metabolic cooperation among commensal bacteria supports Drosophila juvenile growth under nutritional stress. iScience 2020 ; 23 : 101232.

7. Consuegra J, Grenier T, Baa-Puyoulet P, et al. Drosophila-associated bacteria differentially shape the nutritional requirements of their host during juvenile growth. PLoS Biol $2020 ; 18$ : e3000681.

8. Bing X, Gerlach J, Loeb G, et al. Nutrient-dependent impact of microbes on Drosophila suzukii development. mBio 2018 ; 9.

9. Mishra D, Thorne N, Miyamoto C, et al. The taste of ribonucleosides: novel macronutrients essential for larval growth are sensed by Drosophila gustatory receptor proteins. PLoS Biol 2018 ; 16 : e2005570.

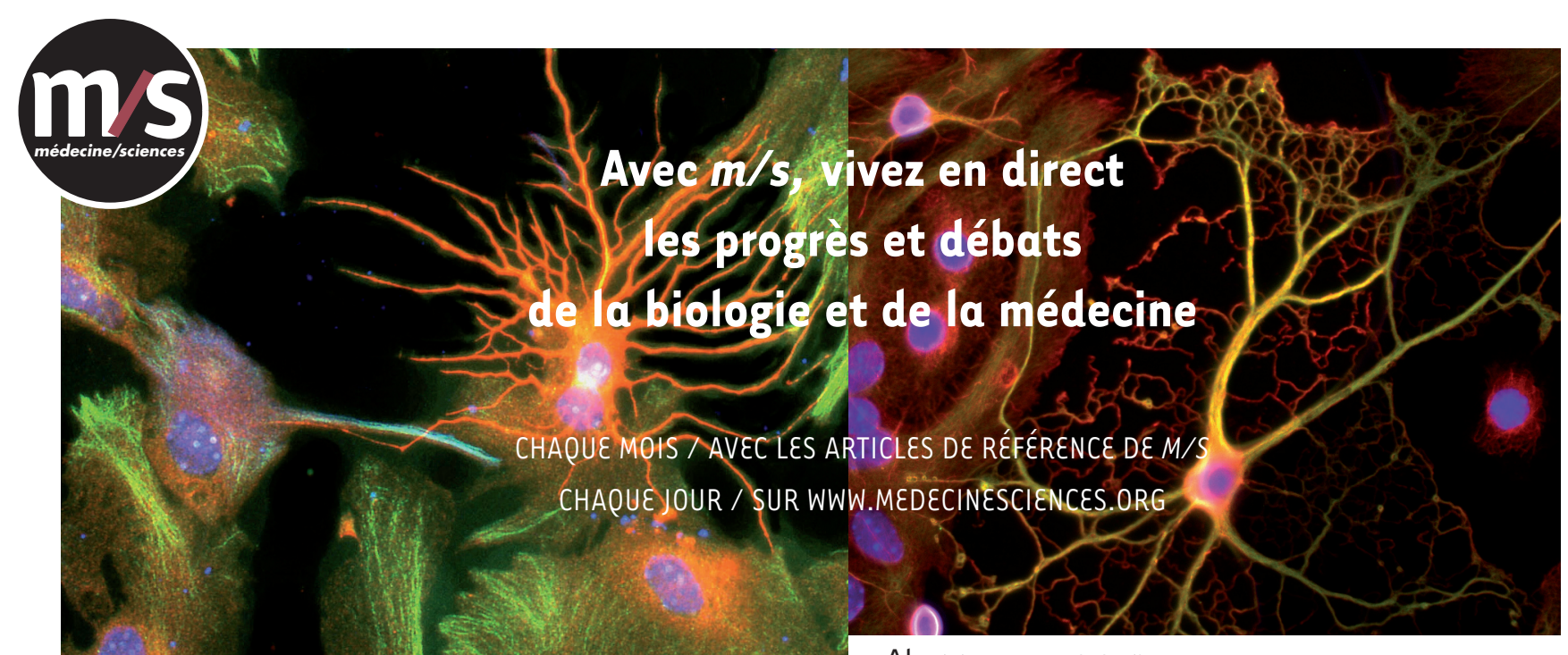

Abonnez-vous sur

WWW.medecinesciences.org 